\title{
Determination of Apoptosis Through Bax Expression in Cryptorchid Testis: an Experimental Study
}

\author{
Mehmet DÜNDAR, ${ }^{1}$ Izzet KOÇAK, ${ }^{1}$ Nil ÇULHACI, ${ }^{2}$ Haluk EROL ${ }^{1}$
}

${ }^{1}$ Department Urology and ${ }^{2}$ Pathology, Medical School, Adnan Menderes University, Aydin, Turkey

The aim was to determine the expression of Bax in germ cells of rats with unilateral experimental cryptorchidism, and to evaluate the role of apoptosis in germ cell loss. Twenty-one prepubertal rats were randomly subdivided into three groups after the execution of the left cryptorchidism model. Group 1 $(n=8)$, group $2(n=6)$ and group $3(n=7)$ rats were killed at the end of the first, second and third month, respectively. Bax expression was assessed in Sertoli cells, spermatogonia and spermatocytes by immunohistochemistry. Percentages of Bax expression in spermatocytes and spermatogonia were decreased in the left testicles in the $2^{\text {nd }}$ and $3^{\text {rd }}$ months compared to results obtained in the $1^{\text {st }}$ month $(\mathrm{p}<0.05)$. Percentage of Bax expression in the left testicles of group 1, at the level of both spermatogonia and spermatocytes was higher than that in the right one $(\mathrm{p}<0.05)$. However, in groups 2 and 3 , the higher Bax expression on the left side was only seen in the spermatocytes $(p<0.05)$. In all groups, the mean weight of the left testicle was lower than that of the scrotal counterpart where the difference was significant only in groups 1 and 3 $(p<0.05)$. The weight of the left and right testicles was increasing with time. In this model of cryptorchidism, the affected testis had a decreased weight compared to the normal one. Based on the increased Bax expression, we think that apoptosis may play a role in the germ cell loss. (Pathology Oncology Research Vol 11, No 3, 170-173)

Key words: Bax, spermatogonia, spermatocyte, undescended testis

\section{Introduction}

Cryptorchidism is one of the most common congenital abnormalities, affecting 1-5\% of men. The histological changes in undescended testis occur up to the age of two. Sperm production depends on the balance between spermatogenesis and apoptosis. Spermatogonia divide 9-11 times and spermatocytes divide twice, thus they constitute a great number of germ cells. Excess germ cells die. Apoptosis or programmed cell death is a physiologic process characterized by morphological and biochemical processes. Loss of the germ cells may also occur under pathological conditions such as deficiency of pituitary hormones or androgens. ${ }^{1}$ Elevated temperature in undescended testis is another cause of germ cell loss. ${ }^{2}$ Heatinduced apoptosis is reported to involve both the mito-

Received: Apr 4, 2005; accepted: Sept 7, 2005

Correspondence: Mehmet DÜNDAR, MD, Adnan Menderes University, Department of Urology, Medical School, 09100 Aydin/ Turkey. Tel.: 0090256 4441256, fax: 0090256 2146495, e-mail: medundar2002@yahoo.com chondria- and endoplasmic reticulum-dependent pathways of apoptosis. ${ }^{3}$ Apoptosis in heat-sensitive germ cells starts with redistribution of Bax from the cytoplasm to the paranuclear area. Initiation of apoptosis is preceded by a redistribution of Bax from a cytoplasmic to paranuclear localization in heat-susceptible germ cells. Such relocation of Bax is further accompanied by sequestration of mitochondria and endoplasmic reticulum (ER) into paranuclear areas, cytosolic translocation of cytochrome $\mathrm{c}$, and is associated with activation of the initiator caspase 9 and the executioner caspases 3, 6, and 7, and cleavage of PARP. ${ }^{4}$ The redistribution of Bax may trigger apoptosis in germ cells. ${ }^{5}$ In contrast to these reports, some other studies did not find any difference in the redistribution of Bax. ${ }^{6}$ Cryptorchidism has proved to cause apoptosis in germ cells. ${ }^{2}$ Zini et al ${ }^{7}$ showed in their experimental study that cryptorchidism results in apoptosis, and orchidopexy reduces the level of apoptosis in undescended testis.

Bax is a pro-apoptotic member of Bcl-2 family, which is necessary for spermatogenesis. In mature animals, deletion of functional Bax results in bulky accumulation of premeiotic germ cells where mature sperm and spermato- 
cytes are almost absent. ${ }^{8}$ Since the mechanism of infertility in cryptorchidism is not known, in this study we intended to evaluate the effect of pro-apoptotic mechanism in germ cell loss through Bax expression, and to determine whether this effect changes in a time-dependent fashion.

\section{Methods}

\section{Experimental animals and model}

This study was approved by the local ethics committee. Three-week-old male Sprague-Dawley rats were obtained from the Veterinary Faculty of Adnan Menderes University. Through an inguinoscrotal approach, experimental left cryptorchidism model was constructed as previously described. In brief, before the testis completed its descent, inguinal canal was found and closed where scrotum would develop. ${ }^{9}$ Xylazine hydrochloride ( $2 \mathrm{mg} / \mathrm{kg}$; Rompun, Bayer, Germany) and ketamine hydrochloride $(10 \mathrm{mg} / \mathrm{kg}$; Ketalar, Parke Davis, Eczacibaşi, Turkey) were given intraperitoneally. Food and water were supplied ad libitum. Group $1(n=8)$, group $2(n=6)$ and group $3(n=7)$ rats were killed at the first, second, and third months, respectively. Both testes were weighed and then fixed in 10\% neutral formaldehyde.

\section{Immunohistochemical examination}

Testes were dissected in a plane perpendicular to the interpolar axis, yielding slices of $1 \mathrm{~mm}$ width. Tissue slices were embedded in paraffin. Paraffin-embedded sections (4- $\mu \mathrm{m}$-thick) were deparaffinized in xylene, rehydrated in alcohol series and immersed in distilled water. The sections were then heated in citrate buffer $(10 \mathrm{mmol} / \mathrm{L}, \mathrm{pH}$ 6.0) for 15 minutes for antigen retrieval. Endogenous peroxidase activity was blocked using a $0.3 \%$ solution of hydrogen peroxide in phosphate buffered saline at room temperature for 10 minutes. Primary antibody against Bax (rabbit polyclonal; Santa Cruz Biotechnology, Santa Cruz, CA) was applied for 60 minutes at room temperature, followed by washing in TRIS buffer. Secondary antibody and streptavidin peroxidase complex were added consecutively for ten minutes each at room temperature and washed in TRIS buffer. Peroxidase activity was visualized with 0.03\% 3,3-diaminobenzidine tetrahydrochloride. Sections were then washed in deionized water, counterstained with Mayer's hematoxylin and mounted. In the evaluation of Bax immunostaining, tonsil tissue was used as control.

All immunostained slides were analyzed by one pathologist in a blinded fashion without knowledge of the data. Both the quantity and quality of immunostaining were assessed in Sertoli cells, spermatogonia and spermatocytes. Immunoreactivity in different types of cells and in different stages was analyzed semiquantitatively according to the following criteria: $(-)$ : no; $(+)$ : weak; $(++)$ : strong; $(+++)$ : very strong immunoreaction. The percentage of
Table 1. Weight of right and left testis in left cryptorchidism model

\begin{tabular}{cccc}
\hline Groups & Right & Left & $p$ \\
\hline $1(\mathrm{n}=8)$ & $0.6288 \pm 0.0332$ & $0.5150 \pm 0.0346$ & $<0.05$ \\
$2(\mathrm{n}=6)$ & $0.9600 \pm 0.1178$ & $0.8950 \pm 0.0878$ & $>0.05$ \\
$3(\mathrm{n}=7)$ & $1.1114 \pm 0.0942$ & $0.9343 \pm 0.1340$ & $<0.05$ \\
\hline
\end{tabular}

Data are expressed as mean \pm SD of testis weight $(\mathrm{g})$

Bax-reactive cells was evaluated with a light microscope by scoring a minimum of 1000 cells in randomly selected areas. The positive cells were counted in 100 adjacent cells in $10 \mathrm{HPFs}$ (high power field), and the total number of positive cells was expressed as a percentage.

Kruskal-Wallis, Wilcoxon and Mann-Whitney U tests were used for statistical analysis.

\section{Results}

The left testicles were lighter than the right ones (Table $1)$. The differences between left and right testes in groups 1 and 3 were significant $(\mathrm{p}<0.05)$.

Cytoplasmic immunostaining of Bax was seen in spermatogonia and spermatocytes, while there was no Bax expression in Sertoli cells (Figures 1,2).

Percentages of germ cells with Bax expression in both spermatocytes and spermatogonia of the left testis were high in the first month (group $1 ; 2.7 \pm 1.9 \%$ and $0.8 \pm 0.2 \%$ ), while they decreased in the second (group $2 ; 0.6 \pm 0.3 \%$ and $0.3 \pm 0.2 \%$ ) and third month (group 3;0.5 $\pm 0.3 \%$ and $0.1 \pm 0.1 \%)$. These differences were statistically significant $(\mathrm{p}<0.05)$, and group 1 values caused the difference. The same evaluation for the right testicles did not reveal any statistically significant difference. With respect to Bax expression percentage in group 1 , significantly more cells stained in the left testis than in the right one, at the level of both spermatogonia and spermatocytes $(\mathrm{p}<0.05)$. In groups 2 and

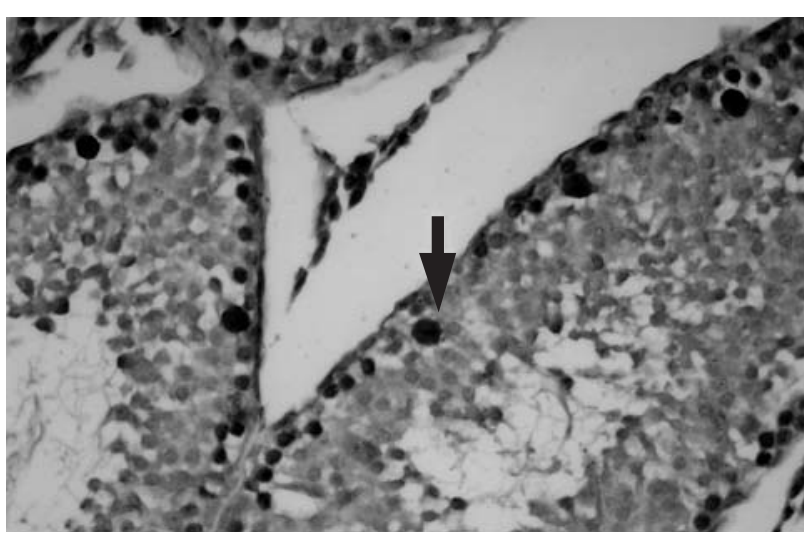

Figure 1. Cytoplasmic Bax immunoreactivity at the spermatogonia level in group 1 ( $x 400)$ 


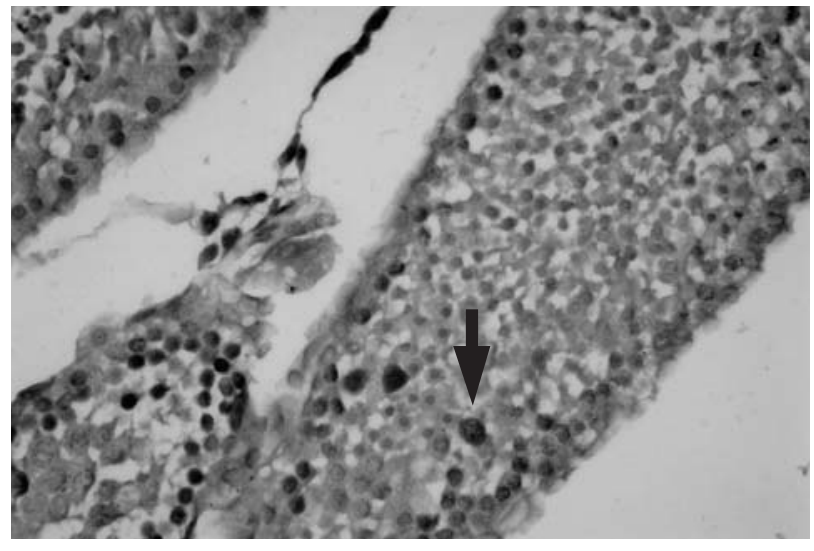

Figure 2. Cytoplasmic Bax immunoreactivity at spermatocyte level in group $3(x 400)$

3 , this was observed only in spermatocytes $(\mathrm{p}<0.05)$, but not in spermatogonia (Table 2). In cryptorchid testes of all groups, the immunoreactivity at the spermatocyte level was strong $(++)$. In spermatogonia and Sertoli cells, immunoreaction was weak (+), and it was similar among the groups and between the left and right testes in the same group.

\section{Discussion}

Cryptorchidism is a common disorder affecting 2-5.8\% of newborns and $0.8 \%$ of infants. ${ }^{10}$ Germ cells undergo apoptosis, the programmed cell death, as shown by morphologic signs and characteristic DNA fragmentation. Apoptosis occurs spontaneously or as a response to changes at the stimulating levels of specific physiologic events. ${ }^{11,12}$

The affected testis in experimental unilateral cryptorchidism has been shown to decrease in weight. ${ }^{2}$ In the study of Tomomasa et $\mathrm{al}^{13}$ on TS inbred rats (congenitally cryptorchid rat), $70 \%$ of which have cryptorchidism at birth, the weight of the affected testis was decreased significantly at the $4^{\text {th }}, 6^{\text {th }}$ and $8^{\text {th }}$ weeks, and spermatogenesis was decreased as well. In their experimental study, Ogi et $\mathrm{al}^{14}$ showed that testicular weight was decreased to $23 \%$ and $31 \%$ on the $4^{\text {th }}$ and $7^{\text {th }}$ days, respectively. In the present experimental study, both testes were found to continue growing where the weight of the left testis was less than that of the right one.

Wang et $\mathrm{al}^{15}$ showed in their experimental unilateral cryptorchidism model that apoptosis increased significantly in the ipsilateral testis. This increase was time dependent. The mostly affected germ cells were spermatocytes. Compared to the normal testis, apoptosis in the contralateral testis was also higher. This was suggested as an explanation for infertility in unilateral cryptorchidism. Tomomasa et $\mathrm{al}^{13}$ also found apoptosis to be prominent particularly in spermatocytes.

While apoptosis in bilateral cryptorchidism may be influenced by changes in systemic factors, unilateral cases are more likely to be affected by local testicular factors. An increase in DNA fragmentation, more prominent in primary spermatocytes, was reported.?

Bcl-2 family members are regulators for apoptosis where Bax promotes cell death. Bax is encountered in Sertoli cells, spermatocytes and spermatogonia. Immunohistochemically, Bax was found to be present in the cytoplasm of Sertoli cells, spermatogonia and spermatocytes, where it was more significant in the final two cell types. The high levels of Bax and Bad in spermatogonia and spermatocytes showed that these cells are more susceptible to apoptosis compared to Sertoli cells. ${ }^{16}$ In the study of Xu et al, ${ }^{17}$ germ cell degeneration induced by cryptorchidism was found to be regulated by apoptosis and dependent on increased testicular temperature. Bcl-2 and Bax proteins were suggested to play a critical role in the apoptosis of male germ cells. In the present study, while the percentage of Bax expression was rising in spermatogonia and spermatocytes over the first month, for the following months it was mainly seen in spermatocytes. This showed that at the first month spermatocyte and spermatogonia, while during the following months spermatocytes were more prone to apoptosis.

In a study using TUNEL method, the damage in spermatogenesis was tracked down to the $4^{\text {th }}$ week. Apoptosis was prominent in spermatocytes, and at the $6^{\text {th }}$ week. ${ }^{13}$ In another study, seminiferous tubules containing apoptotic cells were more intensive in undescended testes. ${ }^{14}$ In their experimental cryptorchidism study on adult mice, Ito et al ${ }^{18}$ demonstrated that apoptosis was prominent in spermatocytes. Wang et $\mathrm{al}^{19}$ evaluated apoptosis in unilateral cryptorchidism model with

Table 2. Time-dependent changes in Bax expression of spermatogonia and spermatocytes, and comparison between left and right testes in each group

\begin{tabular}{|c|c|c|c|c|c|c|c|c|c|}
\hline \multirow[t]{2}{*}{ Germ cells } & \multicolumn{2}{|c|}{$1^{s t}$ month $(n=8)$} & \multirow[b]{2}{*}{$p$} & \multicolumn{2}{|c|}{$2^{\text {nd }}$ month $(n=6)$} & \multirow[b]{2}{*}{$p$} & \multicolumn{2}{|c|}{$3^{\text {rd }}$ month $(n=7)$} & \multirow[b]{2}{*}{$p$} \\
\hline & Right & Left & & Right & $\overline{\text { Left }}$ & & Right & Left & \\
\hline Sperm & $0.1 \pm 0.0$ & $0.8 \pm 0.2$ & S & $0.1 \pm 0.0$ & $0.3 \pm 0.2$ & NS & $0.1 \pm 0.0$ & $0.1 \pm 0.1$ & NS \\
\hline Spermatocyte & $0.2 \pm 0.1$ & $2.7 \pm 1.9$ & $S$ & $0.1 \pm 0.0$ & $0.6 \pm 0.3$ & S & $0.1 \pm 0.0$ & $0.5 \pm 0.3$ & $S$ \\
\hline
\end{tabular}

Data are expressed as percentage of cells (mean \pm SD), S: $p<0.05$ (between right and left testis), NS: not significant 
TUNEL method, and find a significant increase. In all testes affected germ cells were mainly spermatocytes, while Sertoli cells were also affected on the ipsilateral side. In cryptorchidism model, Ohta et $\mathrm{al}^{20}$ observed apoptosis essentially in spermatids and spermatocytes. Many apoptotic cells were phagocytosed by Sertoli cells. They also revealed increased apoptosis in Sertoli and Leydig cells. In the present study we did not find any difference in Bax expression between Sertoli cells of undescended and contralateral testes.

In a clinical study on patients with cryptorchidism, Heiskanen et $\mathrm{al}^{1}$ identified spermatogonia as the main apoptotic cell type in the seminiferous tubules. Apoptosis was shown only in a few Sertoli cells. Apoptosis was more prominent in the normally descended scrotal testis. As an explanation for the decreased number of apoptosis in undescended testis, they suggested the already diminished number of germ cells subjected to apoptosis. Cryptorchidism is a chronic condition starting at birth; thus, such a non-physiologic circumstance may be another explanation for the decreased apoptosis in the affected testis. On the other hand, in experimental cryptorchidism, the affected testis underwent a normal postnatal period, and the acute establishment of the model resulted in the more prominent apoptosis. We observed that in the ipsilateral testis the number of germ cells with Bax expression decreased in a time-dependent fashion. This was in accordance with Heiskanen's explanation; decreasing number of germ cells was subjected to apoptosis in the course of time. Another experimental study by Barqawi et $\mathrm{al}^{21}$ is also in accordance with our results. In this study, it was suggested that in prolonged cryptorchidism the decreased number of germ cells as a result of apoptosis might account for the defect in spermatogenesis.

In our previous study ${ }^{22}$ we observed significant damage in the undescended testis compared to the contralateral one. The effect on the contralateral testis increased in two months. We considered apoptosis as the main mechanism in germ cell death, and it was found to be more significant in spermatocytes and spermatogonia. In the current study, Bax expression was present in both spermatogonia and spermatocytes in the first month, while in the following months this was the case mainly in spermatocytes. The results of this study are in accordance with those in our previous study. ${ }^{22}$

In conclusion, in this study left testicular weights of rats were lower than the ones of the naive contralateral testes. In the affected testis, the number of germ cells expressing Bax, a pro-apoptotic factor, was diminished time-dependently. This can be explained with a lack of germ cells to be exposed to apoptosis, and this absence may be the result of initially increased apoptosis.

\section{Acknowledgements}

This study is supported by the Research Fund of Adnan Menderes University (TPF: 00006).

\section{References}

1. Heiskanen P, Billig H, Toppari J, et al: Apoptotic cell death in the normal and cryptorchid human testis: The effect of human chorionic gonadotropin on testicular cell survival. Pediatr Res 40: 351-356, 1996

2. Shikone T, Billig H, Hsueh JW: Experimentally induced cryptorchidism increases apoptosis in rat testis. Biol Reprod 51: 865-872, 1994

3. Hikim AP, Lue Y, Yamamoto CM, et al: Key apoptotic pathways for heat-induced programmed germ cell death in the testis. Endocrinology 144: 3167-3175, 2003

4. Hikim AP, Lue Y, Diaz-Romero M, et al: Deciphering the pathways of germ cell apoptosis in the testis. J Steroid Biochem Mol Biol 85: 175182,2003

5. Damavandi E, Hishikawa Y, Izumi S, et al: Involvement of Bax redistribution in the induction of germ cell apoptosis in neonatal mouse testes. Acta Histochem Cytochem 35: 449-459, 2002

6. Miura M, Sashagawa I, Suzuki Y, et al: Apoptosis and expression of apoptosis-related genes in the mouse testis following heat exposure. Fertil Steril 77: 787-793, 2002

7. Zini A, Abitbol J, Schulsinger D, et al: Restoration of spermatogenesis after scrotal replacement of experimentally cryptorchid rat testis. Assessment of germ cell apoptosis and eNOS expression. Urology 53: 223-227, 1999

8. Knudson CM, Tung KS, Tourtellote WG, et al: Bax deficient mice with lymphoid hyperplasia and male germ cell death. Science 270: 96-99, 1995

9. Dundar M, Kocak I, Culhaci N: A new experimental model for cryptorchidism: inguinoscrotal approach. Urol Res 29: 178-181, 2001

10. Hellstrom WJG, Monga M: Cryptorchidism and infertility. Male Infertility and Sexual Dysfunction. (Ed: Hellstrom WJG), Springer, 1997, pp 307-322

11. Billig H, Furuta I, Rivier C, et al: Apoptosis in testis germ cells: developmental changes in gonadotropin dependence and localization to selective tubule stages. Endocrinology 136: 5-12, 1995

12. Majno G, Joris I: Apoptosis, oncosis and necrosis. An overview of cell death. Am J Pathol 146: 3-15, 1995

13. Tomomasa H, Adachi Y, Oshio S, et al: Germ cell apoptosis in undescended testis. The origin of its impaired spermatogenesis in the TS inbred rat. J Urol 168: 343-347, 2002

14. Ogi S, Tanji N, Yokoyama M, et al: Involvement of Fas in the apoptosis of mouse germ cells induced by experimental cryptorchidism. Urol Res 26: 17-21, 1998

15. Wang $Z Q$, Todani T, Watanabe A, et al: Germ cell degeneration in experimental unilateral cryptorchidism: role of apoptosis. Pediatr Surg Int 14: 9-13, 1998

16. Yan W, Samson M, Jegou B, Toppari J: Bcl-w forms complexes with $\mathrm{Bax}$ and Bak, and elevated ratios of Bax/Bcl-w and Bak/Bcl-w correspond to spermatogonial and spermatocyte apoptosis in the testis. Mol Endocrinol 14: 682-699, 2000

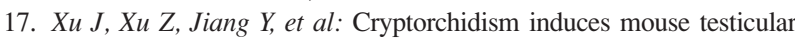
germ cell apoptosis and changes in bcl-2 and Bax protein expression. J Environ Pathol Toxicol Oncol 19: 25-33, 2000

18. Ito K, Tanemura K, Gotoh H, et al: Apoptosis-like cell death in experimentally-induced cryptorchidism in adult mice. J Vet Med Sci 59: 353-359, 1997

19. Wang QZ, Watanabe Y, Toki A, et al: Altered distribution of Sertoli cell vimentin and increased apoptosis in cryptorchid rats. J Pediatr Surg 37: 648-652, 2002

20. Ohta Y, Nishikawa A, Fukazawa Y, et al: Apoptosis in adult mouse testis induced by experimental cryptorchidism. Acta Anat 157: 195204, 1996.

21. Barqawi A, Trummer H, Meacham R: Effect of prolonged cryptorchidism on germ cell apoptosis and testicular sperm count. Asian J Androl 6: 47-51, 2004

22. Kocak I, Dundar M, Hekimgil M, et al: Assessment of germ cell apoptosis on cryptorchid rats. Asian J Androl 4: 183-186, 2002 